\title{
A MULTI-AGENT CONGESTION AND PRICING MODEL
}

\author{
XI ZOU ${ }^{1}$ AND DAVID LEVINSON ${ }^{2}$
}

Received 15 May 2005; received in revised form 4 April 2006; accepted 8 May 2006

\begin{abstract}
A multi-agent model of travelers competing to utilize a roadway in time and space is presented in this paper to illustrate the effect of congestion and pricing on traveler behaviors and network equilibrium. To realize the spillover effect among travelers, $N$-player games are constructed in which the strategy set includes $N+1$ strategies. We solve the $N$-player game (for $N \leq 7$ ) and find Nash equilibria if they exist. This model is compared to the bottleneck model. The results of numerical simulation show that the two models yield identical results in terms of lowest total costs and marginal costs when a social optimum exists.
\end{abstract}

KEYWORDS: Agent-based model, game theory, congestion, queueing, congestion pricing, road pricing

\section{INTRODUCTION}

Congestion is a phenomenon caused by multiple interacting individuals (agents) seeking to use a temporally scarce resource (facility capacity) in a short period of time. On roads, where capacity is scarce relative to the demand that seeks to use it, the facility is called a bottleneck. The consequence of this interaction is queueing. Queues spill back in space and time to affect individuals who may not be seeking the same resource. This study applies a microscopic agent-based approach to understanding congestion that takes the behavior of each road user into consideration. This differs from microscopic analytical models, such as the bottleneck model (Vickery, 1969; Arnott et al., 1990, 1993), with which we compare our model. The agent-based model treats the actions and reactions of road users as a multi-player game in which each user wants to maintain or increase his utility (by reducing cost). In this game, each player (road user) makes decisions on when to start the trip on a roadway that other players also want to use. This decision impacts the journey delay and departure time of other players and thus affects their decisions on when to start the trip. In these interactions, each player suffers costs with probabilities that are determined both by players waiting in queue and by players arriving simultaneously. The major costs are journey delays and penalties for early and late arrival.

Game theory, developed by Von Neumann and Morgenstern (1944) in the 1940s, has been widely applied in the social sciences and in transportation to explain behaviors of agents in conflicts (e.g. airport landing fees (Littlechild and Thompson, 1977), fare evasion and compliance (Jankowski, 1991), truck weight limits (Hildebrand et al., 1990), merging behavior on freeways (Kita et al., 1999), how jurisdictions choose to finance their roads (Levinson, 1999, 2000), aviation (Hansen and Wei, 2001), and the political acceptability of road pricing (Marcucci and Marinim 2003)). de Palma (1992) used a game theory method to study a static route-choice problem. Both Schneider and Weimann, (2004) and Gabuthy et al. (2004) reported on experimental studies of congestion pricing games.

The $N$-player game, in which $N$ is always larger than two, is suitable for our research because the spillover effect (congestion externality) of player interactions on other

\footnotetext{
${ }^{1}$ Department of Civil Engineering, University of Minnesota, 500 Pillsbury DR. SE, Minneapolis, MN 55455, USA.

${ }^{2}$ Department of Civil Engineering, University of Minnesota, 500 Pillsbury DR. SE, Minneapolis, MN 55455, USA. Corresponding author (E-mail: levin031@umn.edu).
} 
players is significant in road traffic and this effect cannot be realized in a two-player game (The words player and agent are used interchangeably). To make the research feasible, we assume all players are rational, meaning they have perfect knowledge of the game and the behaviors of other players, making consistent and optimal decisions for their own benefit. Players may want to improve their utility by changing their strategies. In studying games, we need to know if there are equilibria onto which all players will settle. In game theory, a Nash equilibrium means a strategy combination such that no player can improve his payoff by changing his strategy while all other players keep their strategies unchanged. The expected or average payoffs (costs) in Nash equilibrium must be at least as large (small) as that obtainable by any other strategy. A Nash equilibrium may not yield the lowest total cost for all players, which is rather a system optimal solution. This phenomenon is similar to that illustrated by Wardrop's user equilibrium principle (Wardrop, 1952) used in route choice. With either an explicit cooperation among players or players' knowledge on the history of the game, the total cost may be reduced to a minimum. However, this scenario may not be realized, as travelers cannot be omniscient about the travel decisions of other players in reality, even after repeated learning.

This paper continues Levinson's research (Levinson, 2005) on micro-foundations of congestion and pricing using game theory, by generalizing the formulation of $N$-player games and comparing the results with the analytical bottleneck model. In the next section, we illustrate the $N$-player congestion game for road users. This is followed by the simulation results of the $N$-player congestion game, and a comparison between the game theory model and the bottleneck model. Concluding comments are provided in the last section.

\section{2. $N$-PLAYER GAME}

The number of interacting travelers affects the analysis because the impact of the spillover from some travelers to others could be significant. In this study, we define an $N$-player game as follows:

(1) The number of players is $N$; the player is a traveler driving one vehicle.

(2) Capacity of the transportation facility (which we call a road, but could be any process that can be modeled in a similar way as a server, such as a canal lock or an airport gate), is 1 player per time slot, meaning the deterministic service rate of the system is 1.

(3) For $N$ players, there are $N+1$ arrival strategies. The strategy set is $\left\{e_{N-1}, e_{N-2}, \ldots, e_{1}, o\right.$, $l\} . e_{i}$ denotes arriving early, $o$ denotes arriving on time; $l$ means arriving late.

(4) $N$ players may arrive at the same time, arrive one-by-one in different time slots, or arrive as a pattern that is between these two extreme cases.

(5) If there is no queue, a player traverses the bottleneck with no delay.

In principle, a traveler may arrive at the back of the queue in a period $l_{1}$ or $l_{2}$ (which follow $l$ sequentially) etc., but we exclude those arrival patterns from this analysis for simplicity. 


\section{ARRIVAL PATTERNS}

The arrival patterns are presented in Table 1, which shows the number of arrivals in each time slot. The sum of players in all time slots is $N$. We define the group of players arriving in the same time slot as a cluster. An arrival pattern is a combination of clusters. The number of arrival patterns is very large as described in the following paragraphs.

TABLE 1: Arrival patterns

\begin{tabular}{cccccc}
\hline \hline$e_{N-1}$ & $e_{N-2}$ & $\ldots$ & $e_{1}$ & $o$ & $l$ \\
\hline$N$ & 0 & 0 & $\ldots$ & 0 & 0 \\
$N-1$ & 1 & 0 & $\ldots$ & 0 & 0 \\
& & & $\ldots$ & 1 & 1 \\
1 & 1 & 1 & $\ldots$ & & $N-1$ \\
0 & 0 & 0 & $\ldots$ & 1 & $N$ \\
0 & 0 & 0 & $\ldots$ & 0 & \\
\hline \hline
\end{tabular}

The integers $\{1, \ldots, N\}$ denote the number of players arriving in that time slot; 0 denotes no player arriving in that time slot.

When the number of players is 6 , the formation of arrival patterns is already complicated, as shown in Tables 2 and 3. Table 2 shows all possible arrival patterns or strategies of all players in terms of the $N$-player game just defined in the previous section. Table 3 shows the method of listing and calculating all arrival patterns in the simulation. We ignore vehicle identification for the purpose of simplification. The total number of arrival patterns without considering vehicle identification is $\left(\begin{array}{l}7 \\ 1\end{array}\right)+5 \cdot\left(\begin{array}{l}7 \\ 2\end{array}\right)+10 \cdot\left(\begin{array}{l}7 \\ 3\end{array}\right)+10 \cdot\left(\begin{array}{l}7 \\ 4\end{array}\right)+5 \cdot\left(\begin{array}{l}7 \\ 5\end{array}\right)+\left(\begin{array}{l}7 \\ 6\end{array}\right)=924$

TABLE 2: Example of arrival patterns $N=6$

\begin{tabular}{|c|c|c|c|c|c|c|c|c|c|c|c|}
\hline$e_{5}$ & $e_{4}$ & $e_{3}$ & $e_{2}$ & $e_{1}$ & $O$ & $l$ & $l_{1}$ & $l_{2}$ & $l_{3}$ & $l_{4}$ & $l_{5}$ \\
\hline \multirow[t]{6}{*}{$n_{0}=6 / 0$} & & $\ldots$ & & $\ldots$ & $6 / 0$ & $6 / 0$ & & & & & \\
\hline & & $n_{1}$ & $n_{2}$ & & & & & & & & \\
\hline & $n_{1}$ & $n_{2}$ & $n_{3}$ & & & & & & & & \\
\hline & & $\ldots$ & & $\ldots$ & & & & & & & \\
\hline & & $\cdots$ & & $\cdots$ & & & & & & & \\
\hline & $n_{1}=1$ & $\ldots$ & & $\ldots$ & & $n_{6}=1$ & & & & & \\
\hline
\end{tabular}

$\overline{\sum_{i} n_{i}=6 \text {. This table illustrates all possible arrival patterns, which range from all } 6 \text { players arriving together }}$ (as the first line in the table) to all arriving in different time slots. 6/0 in the table means either 6 players arrive at this time slot or no one arrives.

More generally, the number of arrival patterns is the summation of the number of ways of grouping $N$ players multiplied by the number of combinations in $(N+1)$ time slots, which is:

$$
A=\sum_{i=1}^{N} G_{i}^{N} \cdot\left(\begin{array}{c}
N+1 \\
i
\end{array}\right),
$$

where $G_{i}^{N}$ is the number of patterns that make $i$ groups in $N$ players. The series of $G_{i}^{N}$ is generated as: 
TABLE 3: Number of arrival patterns $N=6$

\begin{tabular}{|c|c|c|c|}
\hline $\begin{array}{c}\text { Number of } \\
\text { arrival clusters }\end{array}$ & $\begin{array}{c}\text { Possible combination of } \\
\text { clusters }\end{array}$ & $\begin{array}{l}\text { Calculation of numbers of arrival patterns } \\
\text { (considering vehicle identifications) }\end{array}$ & $\begin{array}{c}\text { Number of } \\
\text { arrival patterns }\end{array}$ \\
\hline 1 & $\{6\}$ & $\left(\begin{array}{l}7 \\
1\end{array}\right)\left(\begin{array}{l}6 \\
6\end{array}\right)$ & 7 \\
\hline 2 & $\begin{array}{c}\{1,5\},\{5,1\},\{2,4\},\{4,2\}, \\
\{3,3\}\end{array}$ & $\left(\begin{array}{l}7 \\
2\end{array}\right)\left\{2 \cdot\left(\begin{array}{l}6 \\
1\end{array}\right)+2 \cdot\left(\begin{array}{l}6 \\
2\end{array}\right)+\left(\begin{array}{l}6 \\
3\end{array}\right)\right\}$ & 1302 \\
\hline 3 & $\begin{aligned}\{1,1,4\}, & \{1,4,1\},\{1,2,3\} \\
\{1,3,2\}, & \{2,1,3\},\{2,3,1\} \\
\{2,2,2\}, & \{3,1,2\},\{3,2,1\} \\
& \{4,1,1\}\end{aligned}$ & $\begin{array}{l}\left(\begin{array}{l}7 \\
3\end{array}\right)\left\{2 \cdot\left(\begin{array}{l}6 \\
1\end{array}\right)\left(\begin{array}{l}5 \\
1\end{array}\right)+2 \cdot\left(\begin{array}{l}6 \\
1\end{array}\right)\left(\begin{array}{l}5 \\
2\end{array}\right)+2 \cdot\left(\begin{array}{l}6 \\
2\end{array}\right)\left(\begin{array}{l}4 \\
1\end{array}\right)+\cdots\right. \\
\left.+\left(\begin{array}{l}6 \\
2\end{array}\right)\left(\begin{array}{l}4 \\
2\end{array}\right)+2 \cdot\left(\begin{array}{l}6 \\
3\end{array}\right)\left(\begin{array}{l}3 \\
1\end{array}\right)+\left(\begin{array}{l}6 \\
4\end{array}\right)\left(\begin{array}{l}2 \\
1\end{array}\right)\right\}\end{array}$ & 18900 \\
\hline 4 & $\begin{array}{l}\{1,1,1,3\},\{1,1,3,1\}, \\
\{1,3,1,1\},\{3,1,1,1\}, \\
\{1,1,2,2\},\{1,2,2,1\}, \\
\{1,2,1,2\},\{2,2,1,1\}, \\
\{2,1,2,1\},\{2,1,1,2\},\end{array}$ & $\begin{array}{l}\left(\begin{array}{l}7 \\
4\end{array}\right)\left\{3 \cdot\left(\begin{array}{l}6 \\
1\end{array}\right)\left(\begin{array}{l}5 \\
1\end{array}\right)\left(\begin{array}{l}4 \\
1\end{array}\right)+\left(\begin{array}{l}6 \\
1\end{array}\right)\left(\begin{array}{l}5 \\
1\end{array}\right)\left(\begin{array}{l}4 \\
2\end{array}\right)+\cdots\right. \\
+\left(\begin{array}{l}6 \\
1\end{array}\right)\left(\begin{array}{l}5 \\
2\end{array}\right)\left(\begin{array}{l}3 \\
1\end{array}\right)+\left(\begin{array}{l}6 \\
1\end{array}\right)\left(\begin{array}{l}5 \\
2\end{array}\right)\left(\begin{array}{l}3 \\
2\end{array}\right)+\left(\begin{array}{l}6 \\
2\end{array}\right)\left(\begin{array}{l}4 \\
1\end{array}\right)\left(\begin{array}{l}3 \\
1\end{array}\right)+\cdots \\
\left.+\left(\begin{array}{l}6 \\
2\end{array}\right)\left(\begin{array}{l}4 \\
1\end{array}\right)\left(\begin{array}{l}3 \\
2\end{array}\right)+\left(\begin{array}{l}6 \\
2\end{array}\right)\left(\begin{array}{l}4 \\
2\end{array}\right)\left(\begin{array}{l}2 \\
1\end{array}\right)+\left(\begin{array}{l}6 \\
3\end{array}\right)\left(\begin{array}{l}3 \\
1\end{array}\right)\left(\begin{array}{l}2 \\
1\end{array}\right)\right\}\end{array}$ & 54600 \\
\hline 5 & $\begin{array}{c}\{1,1,1,1,2\},\{1,1,1,2,1\} \\
\{1,1,2,1,1\},\{1,2,1,1,1\} \\
\{2,1,1,1,1\}\end{array}$ & $\left(\begin{array}{l}7 \\
5\end{array}\right) \cdot 2 \cdot\left(\begin{array}{l}6 \\
1\end{array}\right)\left(\begin{array}{l}5 \\
1\end{array}\right)\left(\begin{array}{l}4 \\
1\end{array}\right)\left(\begin{array}{l}3 \\
1\end{array}\right)$ & 37800 \\
\hline 6 & $\{1,1,1,1,1,1\}$ & $\left(\begin{array}{l}7 \\
6\end{array}\right) \cdot\left(\begin{array}{l}6 \\
1\end{array}\right)\left(\begin{array}{l}5 \\
1\end{array}\right)\left(\begin{array}{l}4 \\
1\end{array}\right)\left(\begin{array}{l}3 \\
1\end{array}\right)\left(\begin{array}{l}2 \\
1\end{array}\right)$ & 5040 \\
\hline
\end{tabular}

Note: if there are 2 arrival clusters and $N=6$, one slot has 1 agent, the other slot has 5 , (denoted $\{1,5\}$ ) or one slot has 2 agents, and the other has $4(\{2,4\})$, and so on.

$$
\begin{aligned}
& G_{1}^{N}=1, \\
& G_{2}^{N}=\sum_{i=1}^{N-1} G_{1}^{N-i}=N-1, \\
& G_{3}^{N}=\sum_{i=1}^{N-2} G_{2}^{N-i}=\frac{(N-1)(N-2)}{2}, \\
& G_{4}^{N}=\sum_{i=1}^{N-3} G_{3}^{N-i}, \\
& \ldots \\
& G_{N}^{N}=1, \\
& G_{m}^{N}=\sum_{i=1}^{N-m+1} G_{m-1}^{N-i}, \quad 1<m<N .
\end{aligned}
$$

This number $A$ increases exponentially with $N$. For instance, it is 70 for a 4-player game, and 252 for a 5-player game, as noted above 924 for a 6-player game, and 3432 for a 7-player game.

The generation of arrival patterns is crucial in simulation. The first step generates all combinations of numbers that sum to $N$. The second step lists all permutations of these combinations in $(N+1)$ slots. The third step clears all repeated patterns in the list. These 
three steps are very computationally expensive. To illustrate, generating all patterns for a 7-player game takes more than 17 hours of computation using code in MATLAB in a Pentium 2.4G Hz PC with 512MB memory. In this paper, we limit the number of agents to 7 .

\section{DEPARTURE PATTERNS}

If all $N$ players arrive very early (before $\{o\}$ ) at the same time, they depart the road one- by-one and suffer delay. Suppose they are early enough so that the last one arrives and departs on-time. Denote their departure time by $\left\{e_{N-1}, e_{N-2}, \ldots, e_{1}, o\right\}$. If all $N$ players arrive late in $\{l\}$, they impose delays on each other and depart in $\left\{l, l_{1}, \ldots, l_{N-1}\right\}$. Generally combinations of all the departure patterns can be represented as shown in Table 4.

TABLE 4: Combinations of all departure patterns

\begin{tabular}{ccccccccc}
\hline \hline$e_{N-1}$ & $e_{N-2}$ & $\ldots$ & $e_{1}$ & $o$ & $l$ & $l_{1}$ & $\ldots$ & $l_{N-1}$ \\
\hline 1 & 1 & $\ldots$ & 1 & $1_{0}$ & $0_{1}$ & $\ldots$ & 0 & 0 \\
1 & 1 & $\ldots$ & 1 & $0_{0}$ & $1_{1}$ & $\ldots$ & 0 & 0 \\
& & $\ldots$ & & & & $\ldots$ & & \\
0 & 0 & $\ldots$ & 0 & $0_{0}$ & $1_{1}$ & $\ldots$ & 1 & 1 \\
\hline \hline
\end{tabular}

$\overline{\text { Here } 1 \text { denotes one of the players departing in that time slot without considering the vehicle identification, }}$ while 0 denotes that no player departs in that time slot; the subscripts $\{o\}$ and $\{l\}$ denote the time ticks.

Using the 5-player game as an example, the possible departure time slots are $\left\{e_{4}, e_{3}, e_{2}\right.$, $\left.e_{1}, o, l, l_{1}, l_{2}, l_{3}, l_{4}\right\}$. We set up a tag X between on-time $\{o\}$ and late $\{l\}$ to indicate the possible arrival interval, meaning players cannot choose to arrive in the intervals after the $\mathrm{X}$ tag. The departure patterns may include:

All agents depart early or on-time: $\quad\{1,1,1,1,1, \mathrm{X}, 0,0,0,0,0\}$

One agent departs late or very late: $\{1,1,1,1,0, X, 1,0,0,0,0\},\{1,1,1,0,1, X, 1,0,0,0,0\}, \ldots$, $\{0,1,1,1,1, X, 1,0,0,0,0\} ; \ldots$;

$\{1,1,1,1,0, X, 0,1,0,0,0\}, \ldots,\{0,0,1,1,1, X, 1,1,0,0,0\}$

Two agents departs late or very late: $\{1,1,1,0,0, X, 1,1,0,0,0\},\{1,1,0,1,1, X, 1,1,0,0,0\}, \ldots$ $\ldots$

All depart late:

$\{0,0,0,0,0, X, 1,1,1,1,1\}$

Because the last arrival time is $\{l\}$, the players departing in this time slot will depart one-by-one closely while suffering both journey and schedule delays. Thus the departure pattern after the tag X must be "compact" (meaning players depart one-by-one with no unused time slots), unlike patterns before the tag X which could be "loose" (meaning that in some time slots between departures, no player departs). In this example, the possible patterns after tag $X$ include: $\{\ldots, 0 / 1, X, 0,0,0,0\}, \quad\{\ldots, 1, X, 1,0,0,0\}$, $\{\ldots, 1, X, 1,1,0,0\},\{\ldots, 1, X, 1,1,1,0\},\{\ldots, 1, X, 1,1,1,1\}$, in which $0 / 1$ means it could be either 1 or 0 in this time slot. The numbers of possible arrival patterns are: $\left(\begin{array}{l}6 \\ 5\end{array}\right)=\left(\begin{array}{l}5 \\ 5\end{array}\right)+\left(\begin{array}{l}5 \\ 4\end{array}\right),\left(\begin{array}{l}5 \\ 3\end{array}\right),\left(\begin{array}{l}5 \\ 2\end{array}\right),\left(\begin{array}{l}5 \\ 1\end{array}\right)$, and $\left(\begin{array}{l}5 \\ 0\end{array}\right)$ respectively.

This result can be extended to the $N$-player case. The possible patterns and the respective number of possible departure patterns after tag $\mathrm{X}$ are:

$$
\left\{\ldots, 1(0), \mathrm{X}, 0_{1}, \ldots, 0_{N-1}\right\} \quad \Rightarrow\left(\begin{array}{c}
N+1 \\
N
\end{array}\right)=\left(\begin{array}{l}
N \\
N
\end{array}\right)+\left(\begin{array}{c}
N \\
N-1
\end{array}\right)
$$




$$
\begin{aligned}
& \left\{\ldots, 1(0), \mathrm{X}, 1,0_{2}, \ldots, 0_{N-1}\right\} \Rightarrow\left(\begin{array}{c}
N \\
N-2
\end{array}\right) \\
& \left\{\ldots, 1(0), \mathrm{X}, 1,1,0_{3}, \ldots, 0_{N-1}\right\} \Rightarrow\left(\begin{array}{c}
N \\
N-3
\end{array}\right) \\
& \left\{\ldots, 1(0), \mathrm{X}, 1_{1}, 1_{2}, \ldots, 1_{N-1}\right\} \Rightarrow\left(\begin{array}{c}
N \\
0
\end{array}\right)
\end{aligned}
$$

The subscripts of $1 \mathrm{~s}$ and 0 s indicate the time tick. (0) denotes that a zero may be present in that position. The total number of departure patterns is:

$$
D=\sum_{i=0}^{N}\left(\begin{array}{c}
N \\
i
\end{array}\right)=\left(\begin{array}{l}
N \\
N
\end{array}\right)+\left(\begin{array}{c}
N \\
N-1
\end{array}\right)+\cdots+\left(\begin{array}{c}
N \\
0
\end{array}\right)
$$

\section{SOLVING AN N-PLAYER ARRIVAL PATTERN}

The algorithm for solving an $N$-player arrival pattern will:

(1) find the corresponding departure pattern;

(2) estimate probabilities of departure for each player in the departure pattern;

(3) calculate the journey delay;

(4) compute the expected cost for one player in a cluster; and

(5) sum the total cost of all players.

To start with, we assume:

If there is no standing queue, the player who arrives within a cluster departs with a uniform probability within the duration that begins in the time slot when the cluster arrives and ends at the time slot in which the last player of this cluster departs. The probability of a player who arrives in a time slot and departs in a certain time slot is $1 / \mathrm{m}$, where $m$ is the number of members of the cluster to which the player belongs. If there is a standing queue, the first member of the cluster to depart does so when the standing queue has cleared.

The calculation of costs requires the knowledge of arrival patterns and their probability distributions. The expected cost of a player is: $E(s)=\sum_{t} P_{t} \Pi_{t}$, where $P($.) denotes departure probability for a player in a time slot, $1=\sum_{t} P_{t}=P\left(e_{N-1}\right)+\ldots+P\left(e_{1}\right)$ $+P(o)+P\left(l_{1}\right)+\ldots+P\left(l_{N-1}\right) ; \prod_{t}(E, D, L)$ denotes the penalty function for a player; $E$ denotes the unit cost of arriving early; $D$ the unit cost of journey delay; and $L$ the unit cost of arriving late.

The problem of searching Nash equilibria in an $N$-player game is complicated and always affected by the efficiency and numerical stability of algorithms. The algorithms for solving $\mathrm{N}$-player games with a large strategy space usually only approximate the Nash equilibria, which may be close enough to the real one. In this research, an exhaustive, effective, and numerically stable searching algorithm is used. In this algorithm, each departure pattern is tested to see whether it is a Nash equilibrium. If a player changes strategy, while all other players keep their strategies unchanged, his payoff might change. If his cost is reduced after the change, the current strategy combination is not a Nash equilibrium. Otherwise (meaning his payoff does not change, or worsens), the algorithm tests the next player. If the algorithm cannot find a player with decreased payoff in the test, this strategy combination is a Nash equilibrium (NE). The method for recognizing NE and calculating Lowest Total Cost (LTC) is illustrated 
in Figure 1. In the example of a 3-player game in which the pricing policy is $[E D L]=$ [3 04 4], a total of 20 arrival patterns exist. The total cost for each arrival pattern is calculated (blue circles with the left axis). As one can see, five of them achieve the lowest total cost - \$7. Meanwhile, in two arrival patterns (\#18 and \#19), all three players reach Nash Equilibria (red squares with the right axis). One of them (\#18) has the lowest total cost. So we find the NE for this pricing policy.

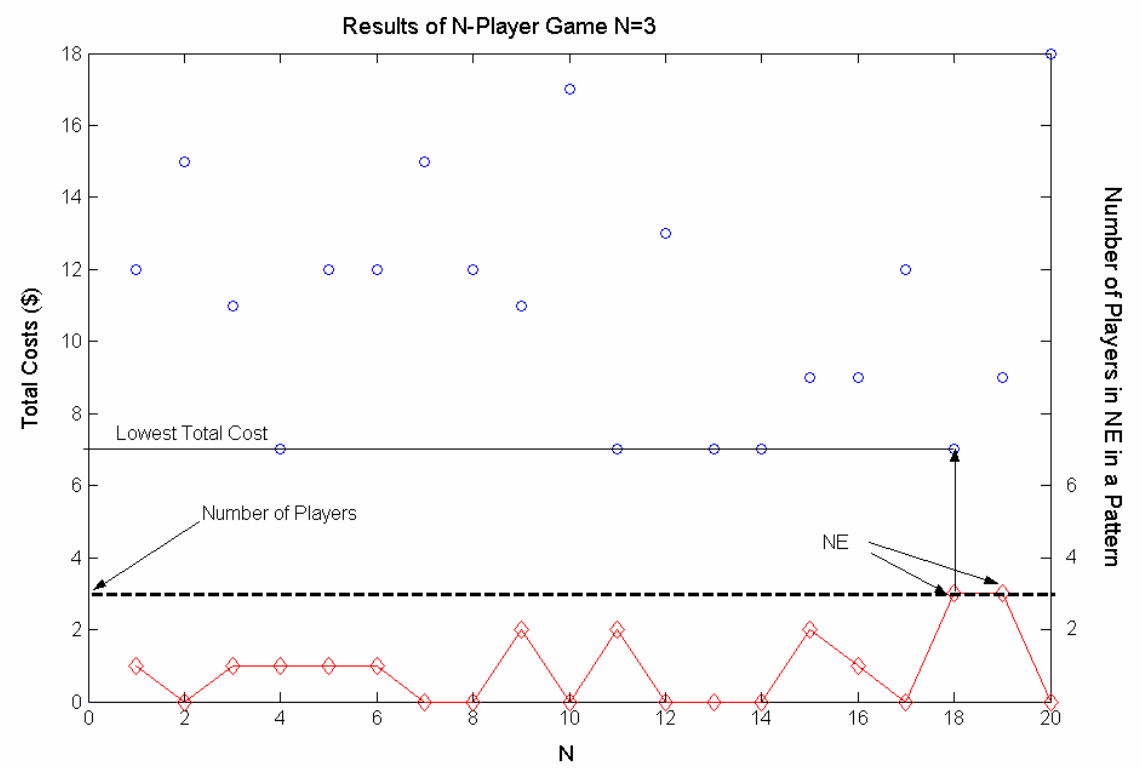

FIGURE 1: Algorithm for recognizing Nash equilibria and calculating the LTC

\section{SIMULATION RESULTS}

After listing all possible departure patterns in an $N$-player game, the simulation calculates the cost suffered by each player in each pattern. An algorithm is used to change the strategy of each player, recalculate costs and discover how many players can reduce their costs while others' strategies remain unchanged. If this number equals zero, this pattern is recognized as a Nash equilibrium. The LTC is obtained by comparing total costs of all patterns. The LTC for all Nash equilibria is also recorded.

Table 5 lists the number of Nash equilibria and the LTCs in $N$-player games. Several penalty patterns are tested, which characterize the results of the $N$-player game. As can be seen, when only one of the three costs (early, delay or late penalties) is suffered by players, the total cost can be as low as zero because players can always find some arrival patterns such that none of them will suffer a cost. When two of the costs apply, the number of Nash equilibria reduces. This trend is more evident when the number of players increases. For instance, when $E=1, D=1$ and $L=0$, there is no NE in games with 5 , 6 , or 7 players. 
TABLE 5: Number of Nash equilibria and lowest total costs in $N$-player games

\begin{tabular}{|c|c|c|c|c|c|c|c|c|c|c|c|c|}
\hline \multirow[t]{2}{*}{$E D L$} & \multicolumn{2}{|c|}{$N=2$} & \multicolumn{2}{|c|}{$N=3$} & \multicolumn{2}{|c|}{$N=4$} & \multicolumn{2}{|c|}{$N=5$} & \multicolumn{2}{|c|}{$N=6$} & \multicolumn{2}{|c|}{$N=7$} \\
\hline & & LTC & & LTC & & LTC & & LTC & & LTC & $\mathrm{\# N}$ & LTC \\
\hline $0,0,0$ & 6 & 0 & 20 & 0 & 70 & 0 & 252 & 0 & 924 & 0 & 3432 & 0 \\
\hline $0,0,1$ & 2 & 0 & 5 & 0 & 14 & 0 & 42 & 0 & 132 & 0 & 429 & 0 \\
\hline $0,1,0$ & 3 & 0 & 4 & 0 & 5 & 0 & 6 & 0 & 7 & 0 & 8 & 0 \\
\hline $1,0,0$ & 3 & 0 & 4 & 0 & 5 & 0 & 6 & 0 & 7 & 0 & 8 & 0 \\
\hline $1,1,0$ & 1 & 0 & 3 & 1 & 2 & 2 & 0 & 4 & 0 & 6 & 0 & 9 \\
\hline $1,0,1$ & 1 & 1 & 3 & 2 & 2 & 4 & 2 & 6 & 3 & 9 & 3 & 12 \\
\hline $0,1,1$ & 1 & $\mathbf{0}$ & 1 & 0 & 1 & 0 & 1 & 0 & 1 & $\mathbf{0}$ & 1 & $\mathbf{0}$ \\
\hline $1,1,1$ & 3 & 1 or 2 & 2 & 2 & 0 & 4 & 0 & 7 & 0 & 10 & 0 & 14 \\
\hline $1,3,4$ & 1 & 1 & 1 & 3 & 0 & 6 & 0 & 10 & 0 & 14 & 0 & 19 \\
\hline $4,0,3$ & 1 & 3 & 2 & 7 & 1 & 13 & 2 & 21 & 3 & 30 & 3 & 42 \\
\hline $3,0,4$ & 1 & $\begin{array}{c}\mathbf{4} \\
(3)\end{array}$ & 1 & 7 & 2 & 13 & 2 & 21 & 2 & 30 & 3 & 42 \\
\hline $3,1,4$ & 1 & $\begin{array}{c}5 \\
\text { (3) }\end{array}$ & 1 & $\begin{array}{l}\mathbf{1 0} \\
(7)\end{array}$ & 1 & $\begin{array}{c}21 \\
(13)\end{array}$ & 1 & $\begin{array}{c}31 \\
(22)\end{array}$ & 2 & $\begin{array}{c}45 \\
(31)\end{array}$ & 3 & $\begin{array}{c}57 \\
(43)\end{array}$ \\
\hline $4,1,3$ & 1 & $\begin{array}{c}4 \\
(3)\end{array}$ & 2 & $\begin{array}{c}8 \\
(7)\end{array}$ & 1 & $\begin{array}{c}19 \\
(14)\end{array}$ & 2 & $\begin{array}{c}31 \\
(22)\end{array}$ & 2 & $\begin{array}{c}40 \\
(33)\end{array}$ & 2 & $\begin{array}{c}63 \\
(45)\end{array}$ \\
\hline $3.05,5,11.88$ & 1 & 3.05 & 0 & 9.15 & 0 & 18.3 & 0 & 30.18 & 0 & 42.38 & 0 & 57.63 \\
\hline $3,5,12$ & 1 & 3 & 0 & 9 & 0 & 18 & 0 & 30 & 0 & 42 & 0 & 57 \\
\hline
\end{tabular}

When Nash equilibria (NE) exist, the LTC is the lowest total cost of Nash equilibria, which are denoted by boldface; the global minimum, if less than that of NE, is listed in the parentheses; otherwise the LTC is the global minimum of all arrival patterns.

An observation of the simulation results is that a journey delay cost higher than one of the early/late penalties will result in a non-Nash-equilibrium scenario when the number of players is large, e.g. $[E, D, L]=[1,1,1],[1,3,4]$ and $[3,5,12]$. In these cases, Nash equilibra may exist among a sub-group of players, but there are always players that can improve their utility by changing strategies. If one wants to move the system to a Nash equilibrium and/or social optimum, one solution is to reduce the ratio of the journey delay cost to early/late penalties to less than one. In this research, we only find some sufficient conditions for the existence of Nash equilibrium. The necessary conditions for the existence of Nash equilibrium, however, cannot be revealed only by a simulation approach. This is because many possible, empirical or non-empirical, costs patterns need to be tested and only an exhaustive search of them can guarantee the necessary condition.

As the costs of early and late arrival increase, the total cost increases. It is shown that when both $E$ and $L$ are larger than $D$, and $D$ is non-zero, there will be some Nash equilibria yielding total costs that are higher than the lowest total cost of the games. But if $D$ equals zero in these cases, such as when [ $\left.\begin{array}{lll}E & D & L\end{array}\right]$ is [ [ $\left.\begin{array}{lll}4 & 0 & 3\end{array}\right]$ and [ $\left.\begin{array}{lll}3 & 0 & 4\end{array}\right]$, the Nash equilibria will also be the solution with the lowest total cost. That is, the user equilibrium in our experiments coincided with a social optimum when there is no penalty for journey delay, except in the case of two players.

According to empirical research (Small, 1982), the last two penalty patterns are more realistic than other patterns, which suggests that the unit cost of arriving late is greater than that of journey delay and the unit cost of arriving early is less than the other two. That is, $E<D<L$. The values of non-integer cost pattern [3.05 5 11.88] were obtained from Arnott et al. (1993) for comparison. The cost pattern [3 5 12] was tested to see the difference affected by small changes in the cost patterns. As shown in Table 5, the differences are small, which shows that at least in this cost pattern the lowest total cost will follow a stable trend and will not be affected by small changes in costs. However, there are a number of cases where $E<D<L$ is satisfied and no NE results. This hints 
that real world traffic may not result in a NE, which is supported by the randomness of much day to day congestion.

All results of the simulation discussed above are illustrated in Figure 2, which includes all LTCs and TCs when NE exist and differ from social optima. One can see that increases in costs of journey delay, compared to early and late penalties ( $E$ and $L$ ), significantly increase the LTCs of society and eliminate the incentive of players to go to social optima instead of Nash equilibra. A remedy for this problem is to reduce the ratio of journey delay cost to early/late penalties (either by reducing the cost of journey delay or by increasing the early and late penalties) so that players are more likely to move from NE to social optima. This means that the tolling policy should be carefully designed to fulfill the desired goal - social optima, which we will further discuss below.

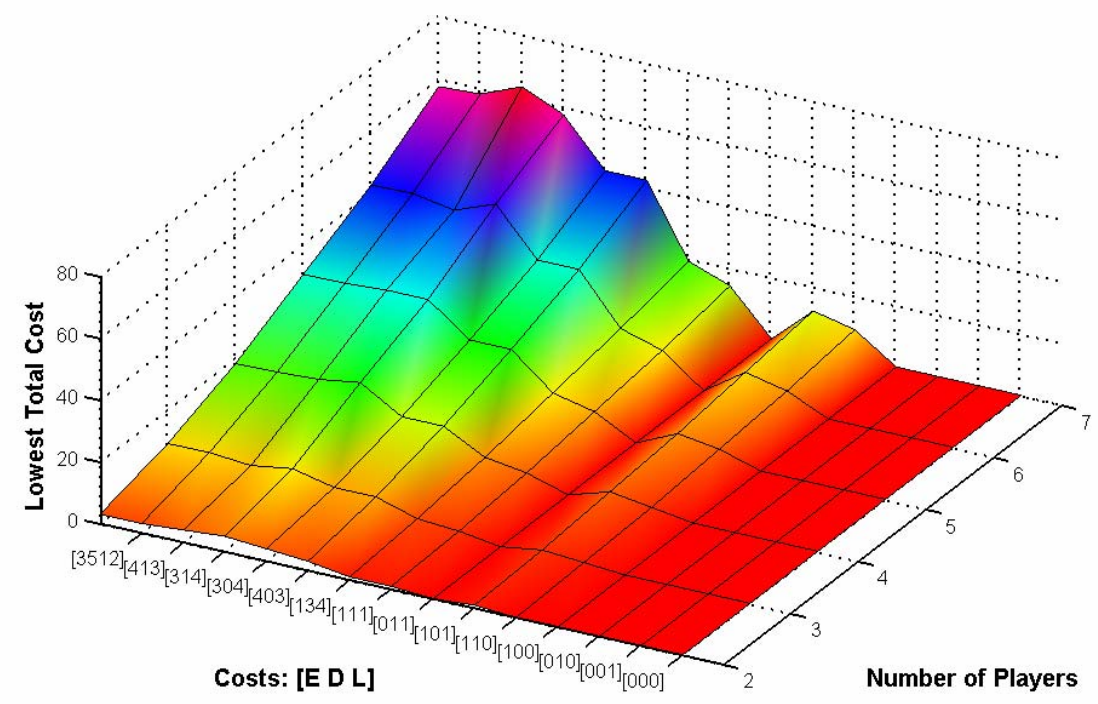

FIGURE 2: Comparison of lowest total costs in $N$-player games

\section{COMPARISON WITH THE BOTTLENECK MODEL}

The bottleneck model of Arnott et al. (1993) addresses different scenarios of road pricing, such as no toll, uniform toll, fine toll and coarse toll. Each has its own total cost (TC), average total cost (ATC) and marginal social cost (MSC). As shown in Table 5, none of the lowest total costs of these games with the cost pattern [3.05 5 11.88] is the result of Nash equilibrium. Instead they are social optima (global minima) among results from all possible arrival patterns. In Arnott et al.’s study, the social optimum can only be achieved in the Fine Toll scenario, in which costs are defined as:

$$
\begin{gathered}
\operatorname{TC}(N, s)=\frac{\Gamma \delta N^{2}}{s}, \\
\operatorname{ATC}(N, s)=\frac{\Gamma \delta N}{s}, \\
\operatorname{MSC}(N, s)=2 \operatorname{ATC}(N, s),
\end{gathered}
$$


where $\delta=E L /(E+L) ; N$ is the number of players; $\mathrm{s}$ is the serving rate, which is defined to be 1 in our research; and $\Gamma=1 / 2$.

Figure 3 summarizes the LTCs in $N$-player games $(\mathrm{N}=2,3,4,5,6,7)$ and the bottleneck model. The figure shows that the results from the two methods are very similar in both the LTC and the MSC (the agent-based model uses Incremental Social Cost (ISC) instead of MSC because of its discrete nature). For the $N$-player game, it is reasonable that no Nash equilibrium exists with the LTC because social optima cannot be guaranteed by user equilibria. Players can often improve their payoffs by changing their strategies, even though the social good may not be improved. For the bottleneck congestion model, the results of the $\mathrm{N}$-player game provide a micro-foundation based on players' behavior. As noted earlier, according to the bottleneck model, the social optimum can be realized by a time varying toll (Fine Toll). In this scenario, there is no queue and thus no cost of journey delay. We checked the arrival patterns corresponding to the LTC in the $N$-player games, and found that they all have the same form as follows:

$\begin{array}{lllllllll}N=3: & \{0 & 1 & 1 & 1 & & & & \\ N=4: & \{0 & 1 & 1 & 1 & 1\} & & & \\ N=5: & \{0 & 1 & 1 & 1 & 1 & 1\} & & \\ N=6: & \{0 & 1 & 1 & 1 & 1 & 1 & 1\} & \\ N=7: & \{0 & 1 & 1 & 1 & 1 & 1 & 1 & 1\}\end{array}$

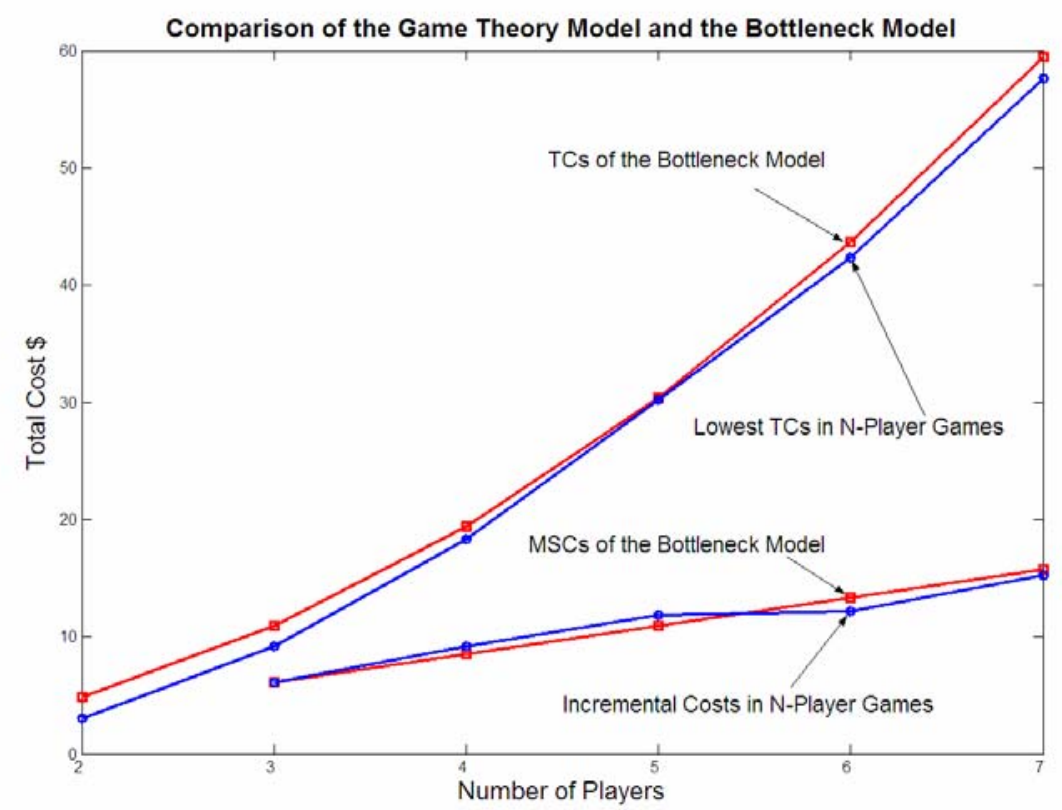

FIGURE 3: Comparisons of the $N$-player game theory model and the bottleneck model

$$
E=3.05, D=5, L=11.88
$$

In these patterns, because all players arrive one by one, there is no queue and no journey delay, as predicted by the bottleneck model.

The game theory approach can help explain how pricing changes players' behavior, and why this leads to the profound differences among the various toll scenarios. In the bottleneck model, the Coarse Toll scenario means the average toll equals average travel 
cost. In this scenario, the only difference with the Fine Toll scenario is $\Gamma=\frac{1}{4}\left(3-\frac{E^{*}(L-D)}{(E+L)^{*}(D+L)}\right)$ (Arnott et al., 1993). We use the penalty pattern [3 14 ] as an example. The results are summarized in Figure 4. As can be seen, the LTCs in $N$ player Games and the TCs of the Bottleneck Model in the Fine Toll scenario are identical with $N=2,3,4,5,6,7$. Further, we compare the TCs of Nash equilibria in the $N$-player games and the TCs of the Bottleneck Model in the Coarse Toll scenario in Figure 4. The two curves are very close, though not totally identical. This implies Nash equilibria can be realized by the Coarse Toll. The results imply consistency between the models, but do not guarantee it.

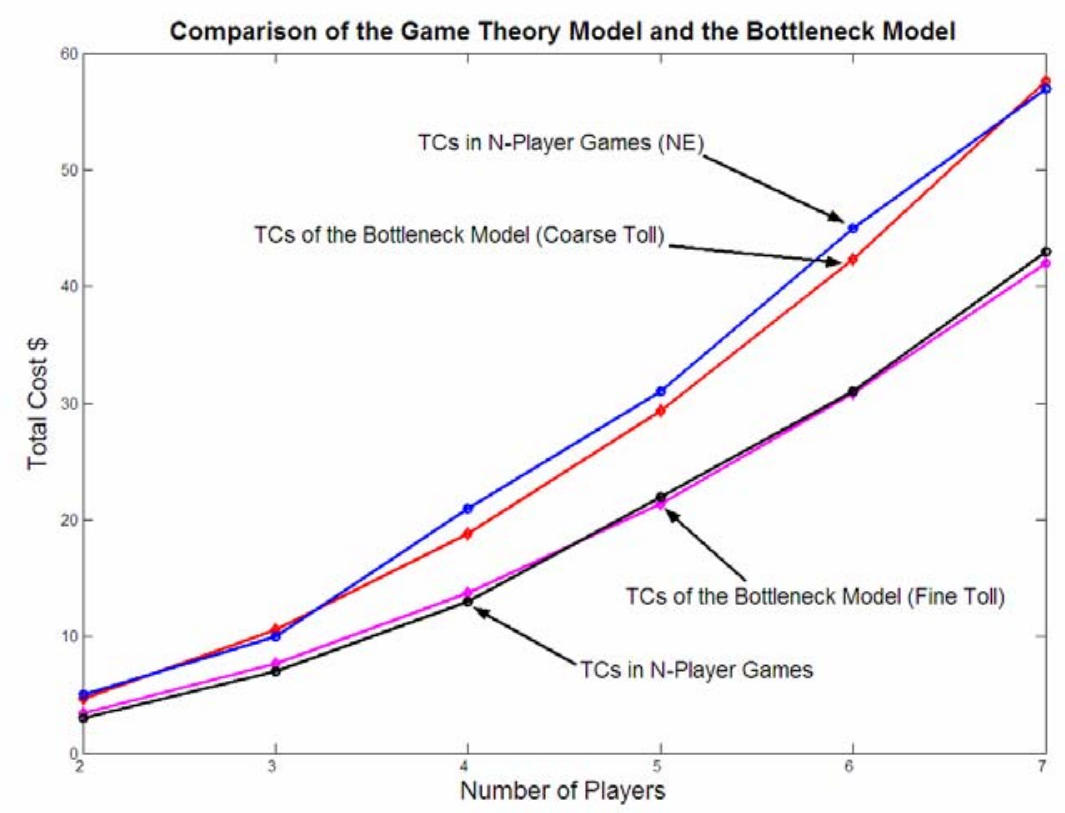

FIGURE 4: Comparisons of the $N$-player game theory model and the bottleneck model

$$
E=3, D=1, L=4
$$

The goal of a network manager is realization of social optima. Our simulation results support Arnott's comparison of Coarse Toll and Fine Toll scenarios by showing that the Fine Toll scenario is close to social optimum, and the Coarse Toll scenario to Nash equilibra. And if management employs a Fine Toll which varies over time rather than just increases fees during peak hours (Coarse Toll), the system will cost less for all players.

There are some other noteworthy findings from the simulation. For instance, the LTC of 3-player games is close to 3 for all cost $E=1$, and $E<D<L$; it is close to 6 in 4-player games; it is close to 10 in 5-player games; it is close to 14 in 6-player games; and it is close to 19 in 7-player games. Multiplying the resulting LTCs for each sized game by 3, we have LTCs of $(9,18,30,42,57)$, which is exactly the result from the cost pattern [3 5 12]. This shows that the LTC is a linear multiple of $E$. As long as $E=1$ and the relation $E$ $<D<L$ obtains, the LTC will be constant. 


\section{CONCLUSIONS}

This paper models traffic congestion as an agent-based phenomenon using $N$-player game theory. The $N$-player game is constructed to simulate the behavior of travelers competing to utilize a roadway (or other capacitated transportation facilities). The simulation presents some interesting results that can be used to illustrate the issues qualitatively. The cost pattern from empirical studies is used to compare with the bottleneck model. The bottleneck model utilizes the average behavior of travelers to illustrate congestion, which overlooks the randomness in travelers' behavior. The game theory approach deals with randomness by assuming a probability function of their decision-making. It is shown that the two results are very similar in terms of lowest total costs and marginal social costs. This provides support for both approaches.

According to the results, there is no user equilibrium under certain cost patterns. In reality, these cost patterns are determined by many social factors that may or may not change significantly over time and space. Thus the resultant traffic pattern, according to the simulation, will not necessarily be a user equilibrium. This is an interesting proposition worth further study.

\section{REFERENCES}

Arnott, R., de Palma, A. and Lindsey, R. (1990) Economics of a bottleneck. Journal of Urban Economics, 27, 111-130.

Arnott, R., de Palma, A. and Lindsey, R. (1993) A structural model of peak-period congestion: a traffic bottleneck with elastic demand. American Economic Review, 83, 161-179.

de Palma, A. (1992) A game-theoretic approach to the analysis of simple congested networks. American Economic Review 82, 494-500.

Gabuthy, Y., Neveu, M. and Boemont, L.D. (2004) Structural model of peak-period congestion: an experimental study. Working paper, BETA-Nancy, University Nancy.

Hansen, M. and Wei, W. (2001) An airline's choice of aircraft size in a competitive environment. Presented at INFORMS Conference Miami, November.

Hildebrand, M., Prentice, B. and Lipnowski, I. (1990) Enforcement of highway weight regulations: a game theoretic model. Journal of the Transportation Research Forum, 30, 442-452.

Jankowski, W.B. (1991) Fare evasion and non-compliance: a game theoretical approach. International Journal of Transport Economics, 38, 275-287.

Kita, H. and Fukuyama, K. (1999) A merging-giveway behavior model considering interactions at expressway on-ramps. In Avishai Ceder (ed.), Transportation and Traffic Theory, Pergamon, pp. 173-187.

Levinson, D. (1999) Tolling at a frontier: a game theoretic analysis. In Avishai Ceder (ed.), Transportation and Traffic Theory, Pergamon, pp. 665-683.

Levinson, D. (2000) Revenue choice on a serial network. Journal of Transport Economics and Policy, 34, 69-98.

Levinson, D. (2005) Micro-foundations of congestion and pricing: a game theory perspective. Transportation Research Part A, 39, 691-704.

Littlechild, S.C. and Thompson, G.F. (1977) Aircraft landing fees: a game theory approach. Bell Journal of Economics, 8, 186-204. 
Marcucci, E. and Marini, M. (2003) Individual uncertainty and the political acceptability of road pricing policies. In J. Schade and B. Schlag (eds.), Acceptability of Transport Pricing Strategies, Pergamon Press, Elsevier Science, pp. 279.

Schneider, K. and Weimann, J. (2004) Against all odds: Nash equilibria in a road pricing experiment. In M. Schreckenberg and R. Selten (eds.), Human Behavior and Traffic Networks, Springer, Berlin, pp. 133.

Small, K.A. (1982) The scheduling of consumer activities: work trips. American Economic Review, 72, 467-479.

Vickery, W. (1969) Congestion theory and transport investment. American Economic Review, 59, 251-260.

Von Neumann, J. and Morgenstern, O. (1944) Theory of Games and Economic Behavior. Princeton University Press, Princeton, NJ, Hildebrand, Marvin, Barry Prentice.

Wardrop, J.G. (1952) Some theoretical aspects of road traffic research. Proceedings of Institution of Civil Engineers, Part II, 1, 325-378. 